\title{
INTERPRETIVE SUMMARIES, AUGUST 2010
}

Invited review: Contemporary environmental issues: A review of the dairy industry's role in climate change and air quality and the potential of mitigation through improved production efficiency. By Place and Mitloehner, page 340\%. Climate change and air quality are issues of growing concern to the general public. The dairy industry's contribution to these environmental problems requires further scientific research because it is not yet fully understood. The development of process-based models that can accurately predict emissions from US dairy production is needed to craft strategies that reduce emissions and to inform regulators.

Use of milk fatty acids composition to discriminate area of origin of bulk milk. By Gaspardo et al., page 341\%. Several studies have shown that milk and milk fat have an important role in the human diet. Some classes of fatty acids, such as n-3, are recognized to be functional compounds that prevent cancer and cardiovascular, inflammatory, and autoimmune diseases. Increasing attention is being paid to adapting the fatty acids profile of milk to obtain a product with positive functions from a human health perspective. The study found that animal diet composition influenced milk fatty acid composition and milk samples were discriminated on the basis of geographical origin.

Identification of ubiquitin in bovine milk and its growth inhibitory effects on human cancer cell lines. By Freiburghaus et al., page 3442. Milk consumption is associated with improved health and reduced risk of diseases such as cancer, heart disease, and diabetes. We identified ubiquitin in bovine milk and studied the effects of the peptide on a human colon cancer cell line. Cells were either untreated or treated with physiologically relevant doses of ubiquitin. Ubiquitin treatment resulted in a reduction of cell proliferation, which became even more apparent after a long treatment time.

Detection of milk powder and caseinates in Halloumi cheese. By Pellegrino et al., page 3453. Halloumi is a traditional cheese of Cyprus that is manufactured from fresh milk. Nevertheless, skim milk powder and caseinates are sometimes illegally added in order to increase cheese yield. These ingredients contain high levels of furosine and lysinoalanine, molecules generated by heating and drying milk protein components. The levels of these 2 molecular markers proved to be strictly correlated in the genuine Halloumi. The confidence interval of this correlation may be adopted as a reliable index of Halloumi cheese genuineness.

Identification of cheese mite species inoculated on Mimolette and Milbenkase cheese through cryogenic scanning electron microscopy. By Melnyk et al., page 3461. The use of mites to ripen cheese is an ancient tradition practiced most commonly in France and Germany. Other than the introduction of new flavors, nothing is known about the chemical, physical, and microbial changes that occur within the cheese caused by the addition of mites. By determining the species of mites present on each cheese sample, further studies will be possible to improve our understanding of the effects they have on the cheese. Cryogenic scanning electron microscopy is used to view the specific features of the mites so identification can be made.

Effect of exopolysaccharide produced by isogenic strains of Lactococcus lactis on half-fat Cheddar cheese. By Costa et al., page 3469. Reduced-fat cheeses possess undesirable textural and flavor characteristics compared with full-fat cheeses. Several approaches have been proposed to overcome these problems, one of which involves the use of exopolysaccharide (EPS)producing starter strains. In this study, the effect of inclusion of an EPS-producing starter on the properties of reduced-fat cheese was investigated using isogenic strains of EPS-producing and non-EPS-producing Lactococcus lactis. Hence, the only difference between the cheeses was the presence of EPS. The results presented help to extend our knowledge of the role of EPS in cheese and how they positively affect the shortcomings of reduced-fat cheeses.

Biochemical patterns in ovine cheese: Influence of probiotic strains. By Albenzio et al., page $348 \%$. This study was undertaken to evaluate the effect of lamb rennet paste containing probiotic strains on biochemical events as proteolysis, lipolysis, and glycolysis of ovine cheese manufactured using starter cultures. Starter cultures coupled with probiotics strains contained in rennet paste affected the acidification and coagulation phases leading to differences in maturing profile of ovine cheese. Use of probiotics in trial cheeses did not adversely impact preference or acceptability; in fact, panelists scored probiotic cheeses higher in preference over traditional cheese, albeit not significantly.

Sensitive real-time measurement of the refractive index and attenuation coefficient of milk and milk-cream mixtures. By Calhoun et al., page $349 \%$. We present the most accurate and sensitive measurements to date of the refractive index and attenuation coefficient of milk and milk-cream mixtures. We demonstrate a novel sensor that makes direct measurements on the samples without the need for any dilutions by observing the reflectance profile of a divergent laser beam made incident on the sample surface. Our sensor is compact, portable, inexpensive, and capable 
of real-time, online monitoring without any mechanical adjustments. We found that the reflectance data is well described by a new model that we developed of total internal reflection from a turbid medium.

Chronic metabolic responses of postpartal dairy cows to subcutaneous glucagon injections, oral glycerol, or both. By Osman et al., page 3505. Fatty liver syndrome, a metabolic disturbance, starts around calving as a consequence of a negative energy balance. Accumulated liver lipids cause the liver to malfunction, which leads to increased vulnerability to other fatty liver-related health disorders. We show that a single daily dose of glycerol for the first $14 \mathrm{~d}$ postpartum can be used to potentiate the preventative effect of multiple once-daily injections of glucagon to alleviate some symptoms of fatty liver syndrome in Holstein dairy cows

Effect of Johne's disease status on reproduction and culling in dairy cattle. By Smith et al., page 3513. Data from 6 commercial US dairy herds over a 3 - to 7-yr period were analyzed to determine the effect of Mycobacterium avium ssp. paratuberculosis (MAP) on time to culling and time to calving. Animals positive for MAP were culled at higher rates than negative animals, and cows shedding high quantities of MAP had longer calving intervals than cows that were ELISA positive only or shedding low quantities of MAP. These results demonstrate that the economic impact of MAP, higher cull rates and longer calving intervals, should be considered when determining optimal control measures.

Sensitivity and specificity of infrared thermography in detection of subclinical mastitis in dairy cows. By Polat et al., page 3525. Mastitis, the most costly disease in dairy operations, occurs at a high prevalence in all countries. Its early diagnosis is crucial for effective treatment and, consequently, for reducing economic losses. Routine diagnostic indicators (California Mastitis Test and somatic cell count) are strongly correlated with the udder skin surface temperature. As a noninvasive and quick method, infrared thermography is able to detect temperature increase on udder skin and can be employed for early diagnosis of subclinical mastitis with as high sensitivity and specificity as California Mastitis Test.

Separate housing for one month after calving improves production and health in primiparous cows but not in multiparous cows. By Ostergaard et al., page 3533. An experiment was conducted in 6 commercial dairy herds to test whether cows housed for 1 mo after calving in a separate group with herdmates would produce more milk and would be healthier than cows integrated in the group of all remaining lactating cows just after calving. Separate housing resulted in increased milk yield in primiparous cows and in fewer primiparous cows being treated for ketosis.

Medium-term effects of repeated exposure to stray voltage on activity, stress physiology, and milk production and composition in dairy cows. By Rigalma et al., page 3542. Stray voltage $(<10 \mathrm{~V})$ is an unpredictable electric phenomenon that may impair animal welfare and production. Seventy-four dairy cows were divided into 3 groups and were exposed to stray voltage $(1.8 \mathrm{~V})$ for $8 \mathrm{wk}$ on their water trough either permanently (predictable), randomly (unpredictable, $36 \mathrm{~h} / \mathrm{wk}$ ), or not at all. Permanent exposure induced a transient acute stress response, whereas unpredictable voltage exposure was considered a mild chronic stressor in dairy cows, with no impairment in production and only slight modifications in stress physiology.

Lying behavior as an indicator of lameness in dairy cows. By Ito et al., page 3553. Lying behavior and gait scores, a measure of lameness, were compared for 1,319 cows on 28 commercial dairy farms. Farms using deep-bedded stalls had lower rates of lameness than farms using mattress stalls. Cows that had very high lying times and long lying bouts were most likely lame. Systems of monitoring lying behavior may be useful for identifying lame cows on farm.

Potassium and insulin affect the contractility of abomasal smooth muscle. By Türck and LeonhardMarek, page 3561. Abomasal displacement is an important disease of dairy cows. Although many factors can be related to its occurrence, the pathogenesis of this disease is still insufficiently understood. The levels of hypokalemia or hyperinsulinemia that occur in cows around parturition are able to reduce the contractility of abomasal muscles in vitro and could contribute to the pathogenesis of abomasal atony and displacement.

Day-to-day variation in milk yield and milk composition at the udder-quarter level. By Forsbäck et al., page 3569. The growing herd size in dairy production requires reliable tools for management of the cows. Automatic in-line sampling and measurement of milk composition is one alternative for monitoring milk quality and health disturbances. This investigation studied day-to-day variations in milk composition and milk yield at udder-quarter and cow composite level in clinically healthy cows. It was concluded that the day-to-day variations in the 4 udder quarters within a healthy cow are similar; thus, within-udder comparisons are useful for detecting disturbances.

Follicular wave of the ovulatory follicle and not cyclic status influences fertility of dairy cows. By Bisinotto et al., page 3578. Anovular cows have reduced 
pregnancy per artificial insemination $(\mathrm{P} / \mathrm{AI})$. When subjected to synchronization protocols, they ovulate the dominant follicle of the first wave at insemination. Cyclic cows initiating the synchronization protocol with low progesterone concentration had similar P/AI to that of anovular cows, and both were less than that of cows initiating the synchronization during diestrus. Cyclic cows induced to ovulate the first wave dominant follicle had reduced P/AI compared with cows ovulating the second wave follicle. Insemination to the first wave follicle, or development of the follicle under low progesterone concentrations, or both, might be mechanisms for reduced fertility in anovular cows.

Milk flow-dependent vacuum loss in high-line milking systems: Effects on milking characteristics and teat tissue condition. By Ambord and Bruckmaier, page 3588. The investigation was conducted to study effects of vacuum stability during the entire milking on milking characteristics, teat conditions, and udder health. A partial compensation of vacuum loss during the period of high milk flow caused an increased peak flow rate, whereas total milk yield and average milk flow did not change. Postmilking teat condition and somatic cell count did not differ with or without vacuum compensation. In conclusion, the compensation of milk flow-dependent vacuum loss during milking increased peak flow rate but did not necessarily result in faster milking or in better teat condition and udder health.

Association between the proportion of sampled transition cows with increased nonesterified fatty acids and $\beta$-hydroxybutyrate and disease incidence, pregnancy rate, and milk production at the herd level. By Ospina et al., page 3595. The herd alarm level was defined as the proportion of sampled animals per herd with increased pre- and postpartum nonesterified fatty acids and $\beta$-hydroxybutyrate concentrations that were associated with negative herd-level outcomes. In a prospective cohort study, 1,672 cows from 60 dairy herds in the northeast United States were used to identify the herd alarm level. If more than $15 \%$ of sampled animals had metabolite concentrations above the defined thresholds, then there was increased disease incidence and decreased pregnancy rate and mature equivalent 305 milk yield. Approximately two-thirds of sampled herds were above the herd alarm level.

Noninvasive assessment of autonomic activity for evaluation of pain in calves, using surgical castration as a model. By Stewart et al., page 3602. Acute autonomic responses, measured by noninvasive methods, were used to evaluate responses to pain following surgical castration of calves. Local anesthetic reduced but did not eliminate pain responses to castra- tion. This study provides insight into the immediate pain responses to castration and describes alternative methods for pain assessment that are less invasive than methods currently used.

In vitro antimicrobial effect and in vivo preventive and therapeutic effects of partially purified lantibiotic lacticin NK34 against infection by Staphylococcus species isolated from bovine mastitis. By Kim et al., page 3610. The preventive and therapeutic effects of lantibiotic lacticin NK34 against in vivo murine infection by Staphylocccus aureus and Staphylococcus simulans were determined after identifying the 2 strains from each group with the greatest lacticin NK34 susceptibility and measuring their minimal lethal dose (MLD) in imprinting control region mice. Mice infected experimentally with $S$. aureus 69 MLD or S. simulans 55 MLD were treated with lacticin NK34. Treated mice demonstrated an $80 \%$ survival rate (48/60 mice) compared with a $7.5 \%$ survival rate (3/40 mice) in distilled water-treated control mice. This data suggested that lacticin NK34 might be useful in the control of bovine mastitis and systemic bacterial infection.

Detection of clinical mastitis with sensor data from automatic milking systems is improved by using decision-tree induction. By Kamphuis et al., page 3616. This paper describes the development and validation of a clinical mastitis detection model using decision-tree induction. Sensor data of 3.5 million quarter milkings were collected from 9 Dutch dairy herds milking automatically and were, if possible, combined with visual observations of clinical mastitis. A decision tree was trained using quarter milkings with $(\mathrm{n}=243)$ or without $(\mathrm{n}=24,717)$ clinical mastitis and validated using 105 quarter milkings with and 50,000 quarter milkings without clinical mastitis. Compared with models currently used by automatic milking systems, the decision tree showed a similar sensitivity (40\%) and a $50 \%$ decrease in false-positive alerts.

Efficacy of formalin, copper sulfate, and a commercial footbath product in the control of digital dermatitis. By Teixeira et al., page 3628. Three major infectious digital diseases are prevalent on North American dairy farms: digital dermatitis, interdigital dermatitis, and foot rot (infectious pododermatitis). The practice of foot bathing using disinfectants such as formalin and copper sulfate is a popular management strategy for controlling infectious digital diseases. A new commercially available footbath product named Dragonhyde (T-Hexx Dragonhyde HBC, Hydromer, Branchburg, NJ) was compared with formalin and copper sulfate foot baths. Dragonhyde footbathing was more effective in the prevention of digital dermatitis than formalin and was as effective as copper sulfate. 
Technical note: Evaluation of a scoring system for rumen fill in dairy cows. By Burfeind et al., page 3635. Feed intake has been identified as potential indicator for early disease detection in dairy cows, but monitoring individual intake on commercial farms remains a challenge. Recent interest has revolved around a 5-point subjective scoring system for rumen fill, but no work to date has validated this as measure of feed intake. Our results indicate that changes in visual rumen fill scores within cow over time may be a reasonable estimate of changes in intake.

Nitrogen utilization, nutrient digestibility, and excretion of purine derivatives in dairy cattle consuming rations containing corn milling coproducts. By Gehman and Kononoff, page 3641. Feeding high levels of corn milling co-products to dairy cows may be limited by perceived complications of feeding rations high in fat and protein. We hypothesized that feeding a combination of wet corn gluten feed and wet distillers grains with solubles would allow for a higher inclusion of co-products than if fed separately. Dry matter intake and nutrient digestibility were improved with the co-product ration, whereas nitrogen excretion in manure and fat-corrected milk were similar to a ration without co-products. This indicates that high coproduct rations can be fed without additional environmental concerns compared with a traditional ration.

Inclusion of psyllium in milk replacer for neonatal calves. 1. Effects on growth, digesta viscosity, rate of passage, nutrient digestibilities, and metabolites in blood. By Cannon et al., page 3652. Based on beneficial effects of psyllium supplementation in other species, newborn dairy calves were fed milk replacer either without or with psyllium supplementation (1.1\% of dry matter). Measurements were made weekly through 28 d of age. Although psyllium supplementation did not affect intakes or growth of calves, viscosity of digesta was increased and digesta passage rate decreased. Dry matter digestibility was slightly lower for the psyllium diet, but blood glucose concentration was more stable in psyllium-supplemented calves. These effects might be beneficial to nutrient use by calves.

Effects of dietary supplementation of methionine and lysine on milk production and nitrogen utilization in dairy cows. By Wang et al., page 3661. Supplementation of lysine or methionine analogs improved the amino acid uptake of the mammary gland in Chinese Holstein dairy cows. Milk yield was increased by supplementing lysine, or methionine, or both, and methionine supplementation increased milk fat content. Lysine supplementation had no effect on milk content of fat or protein. To improve milk performance and nitrogen utilization of dairy cows, the optimal ratio of lysine to methionine was $3: 1$.
Dietary structural to nonfiber carbohydrate concentration during the transition period in grazing dairy cows. By Roche et al., page 3671 . Manipulating the ratio of structural to nonfiber carbohydrate in the prepartum diet of pasture-grazed dairy cows did not affect milk production. In comparison, a postpartum diet high in nonfiber carbohydrates, but comparatively isoenergetic to a low nonfiber carbohydrate diet, resulted in greater milk and milk protein production, whereas milk fat content and yield declined. Body weight and body condition score were not affected by dietary carbohydrate type. Blood metabolites indicated a disconnect between plasma nonesterified fatty acids and $\beta$-hydroxybutyrate, suggesting that the latter may not be an effective indicator of energy balance in pasture-fed cows.

Comparison of fermentation of diets of variable composition and microbial populations in the rumen of sheep and Rusitec fermenters. I. Digestibility, fermentation parameters, and microbial growth. By Martínez et al., page 3684. Rusitec fermenters are a useful tool to study ruminal fermentation, thus reducing the number of animals used in experimentation. This study compared the effects of 4 diets on ruminal fermentation and microbial growth in Rusitec fermenters with those detected in sheep. Fermenters detected similar differences to those found in vivo among diets differing in forage quality for most measured parameters but did not satisfactorily reproduce differences that were observed between diets with different forage to concentrate ratios. Changing operation conditions (e.g., retention time, saliva infusion) in fermenters may allow a better simulation of ruminal fermentation of high-concentrate diets.

Comparison of fermentation of diets of variable composition and microbial populations in the rumen of sheep and Rusitec fermenters. II. Protozoa population and diversity of bacterial communities. By Martínez et al., page 3699. Ruminal microbial populations in sheep were compared with those developed in fermenters simulating the ruminal ecosystem. Fermenters had lower numbers of protozoa and cellulolytic bacteria than sheep fed the same diets, but greater bacterial numbers. Diversity of bacteria in the solid phase was affected by the type of forage in sheep and fermenters, but bacteria in the liquid phase responded to dietary characteristics in a different way within each system. The different conditions in the fermenters compared with sheep rumen might have caused a selection of some bacterial strains.

An evaluation of Brix refractometry instruments for measurement of colostrum quality in dairy cattle. By Bielmann et al., page 3713. The objectives of this study were to evaluate Brix refractometers for 
their effectiveness at determining colostrum quality. Based on the results of this study, a cut-off level of $22 \%$ (or above) Brix units can be used to determine that colostrum from dairy cattle is of good quality. Both the optical and digital Brix refractometers can be used to measure the quality of either fresh or frozen colostrum. Both of these instruments have very good test characteristics using the gold standard radial immunodiffusion assay laboratory test. In summary, the optical and digital Brix refractometers show considerable potential for use as management tools on dairy operations, helping to ensure that calves receive good-quality colostrum.

Effects of timing of corn silage supplementation on digestion, fermentation pattern and nutrient flow during continuous culture fermentation of a short and intensive orchardgrass meal. By Gregorini et al., page 3722. This study investigated digestibility, fermentation, and nitrogen flow during continuous culture fermentation of herbage only and herbage plus corn silage, fed either 1 or $9 \mathrm{~h}$ before a single herbage meal. The results indicate that silage allocation at 9 $\mathrm{h}$ rather than $1 \mathrm{~h}$ before an herbage meal alters the products of digestion and may increase glucogenic nutrient supply to the host animal and improve nitrogen utilization by reducing ammonia nitrogen losses.

Feed delivery method affects the learning of feeding and competitive behavior in dairy heifers. By Greter et al., page 3730. The objective of this study was to determine how different feeding methods may affect the learning of feeding and sorting behavior, as well as feed bunk competition, of growing dairy heifers. Thirty-two Holstein heifers were exposed to one of 2 dietary treatments for $13 \mathrm{wk}$ [total mixed ration (TMR) or top-dressed ration] and were then switched to an unfamiliar TMR for $7 \mathrm{wk}$. Results demonstrated that feeding a TMR to growing dairy heifers from a young age promotes a more even diurnal feeding pattern, minimizes feed bunk competition, and promotes more normal fecal consistency compared with heifers previously fed a top-dressed ration, thus improving the overall welfare of these animals.

Effect of different rumen-inert fatty acids supplemented with a dietary antioxidant on performance and antioxidative status of earlylactation cows. By Wang et al., page 3738. The effect of dietary antioxidants with different fatty acids on antioxidative status and performance in high-lactating cows was evaluated. Cows supplemented with lower saturation fatty acids in the form of Ca salts of longchain fatty acids had lower milk yield compared with those supplemented with higher saturation fatty acids. Supplementation of lower saturation fatty acids resulted in inferior plasma antioxidative status, as indicated by lower plasma superoxide dismutase and higher malon- dialdehyde. The addition of an antioxidant was effective in reducing the negative effect of feeding the lower saturation fatty acids and resulted in improved milk performance and antioxidative status.

Effect of dietary rumen-protected choline on milk production of dairy cows: A meta-analysis. By Sales et al., page 3746. Models were developed to permit the calculation of changes in milk yield and milk composition from amounts of rumen-protected choline chloride in dairy cow diets. This was achieved with a meta-analysis of treatment means from 11 studies. This evaluation presented evidence that the addition of rumen-protected choline chloride to dairy cow diets has a positive effect on milk yield and milk protein content. However, it does not have any influence on milk fat content.

Decrease of ruminal methane production in Rusitec fermenters through the addition of plant material from rhubarb (Rheum spp.) and alder buckthorn (Frangula alnus). By García-González et al., page 3755. Adding rhubarb in small quantities to a diet for ruminants caused a steady decrease in the production of ruminal methane when a rumen simulating fermenter was used as a model. No other relevant effects were observed in ruminal digestion and fermentation, suggesting the inclusion of this plant species or its derivatives in ruminant feeds may involve productive and environmental benefits.

Effects of microbial inoculants on corn silage fermentation, microbial contents, aerobic stability, and milk production under field conditions. By Kristensen et al., page 3764. This study investigated the effect of microbial corn silage inoculants as applied under practical farming conditions on silage fermentation variables and milk production. The use of homofermentative inoculant (Lactobacillus pentosus and Pediococcus pentosaceus) did not have any detectable effects on silage fermentation. Inoculation with heterofermentative lactic acid bacteria (Lactobacillus buchneri) increased the stability of treated silages accompanied by numerous changes in contents of fermentation products and growth of yeast. Treatments were without effects on milk production under practical conditions.

Technical note: Evaluation of procedures for analyzing ration sorting and rumen digesta particle size in dairy cows. By Maulfair and Heinrichs, page 3784. A study was conducted to determine whether taking total mixed ration samples throughout the day changed particle size of the ration and ration sorting behavior of lactating cows. This study also evaluated the effect of squeezing rumen digesta samples through several layers of cheesecloth on particle size distribution determined via wet sieving. Mixing total mixed rations 
several times throughout the day to collect representative samples reduced particle size of total mixed rations and led to the conclusion that cows were sorting less. Squeezing rumen digesta through 4 layers of cheesecloth did not affect particles $>0.15 \mathrm{~mm}$ but reduced rumen fluid-associated dry matter.

Genetic parameters for milk coagulation properties in Estonian Holstein cows. By Vallas et al., page 3789. Milk coagulation is an important process in cheese production. Milk coagulation traits are commonly defined as milk coagulation time and curd firmness. A limited number of studies have reported reliable genetic parameter estimates for milk coagulation traits. In our large-scale study we found substantial genetic variation for milk coagulation traits. Genetic correlations for milk coagulation traits with milk yield and milk composition traits were low, except for correlations of 0.69 between milk coagulation time and $\mathrm{pH}$ and 0.48 between curd firmness and protein percentage.

Effects of $\beta$-א-casein (CSN2-CSN3) haplotypes and $\beta$-lactoglobulin $(B L G)$ genotypes on milk production traits and detailed protein composition of individual milk of Simmental cows. $B y$ Bonfatti et al., page 3797. Effects of milk protein genes on milk production traits, content of protein fractions, and detailed protein composition were estimated. Milk protein genes exerted trivial effects on milk production traits and marked effects on expression of protein fractions. Information on genetic variation of protein composition might be used in selective breeding to enhance milk coagulation properties and cheese yield by increasing content of favorable protein fractions in milk.

Effects of $\beta$-к-casein (CSN2-CSN3) haplotypes, $\beta$-lactoglobulin $(B L G)$ genotypes, and detailed protein composition on coagulation properties of individual milk of Simmental cows. $B y$ Bonfatti et al., page 3809. Effects of protein genes on milk coagulation properties were estimated using models accounting for detailed protein composition. Milk coagulation properties were affected by variation of protein composition attributable to the allele-specific protein expression of $\kappa$-casein and not affected by different molecular properties of $\kappa$-casein protein variants. Conversely, variants of $\beta$-casein had direct effects on the coagulation process. After further investigation of genetic parameters, detailed protein composition might be used in selective breeding to enhance milk coagulation properties.

Multivariate analysis of a genome-wide association study in dairy cattle. By Bolormaa et al., page 3818. The statistical power of detecting associations using multiple-trait genome-wide association study was as good as or better than that of the best single-trait genome-wide association study. Additional associations were detected with use of the multivariate methods. The polymorphisms affecting milk production traits followed 4 pleiotropic patterns. The multivariate analysis did not appear to increase the precision of putative quantitative trait loci mapping.

Exploring polymorphisms and effects of candidate genes on milk fat quality in dairy sheep. By Crisà et al., page 3834. Milk fatty acid profiles play an important role in dairy sheep farming, both as a source of nutraceuticals and as determinants of cheese characteristics. The effect of a large number of single nucleotide polymorphisms within genes involved in the metabolism of milk fat upon the fatty acid composition was evaluated, and some genes that play a role in the desaturation of stearic fatty acid into polyunsaturated fatty acids and in the variability of total fat content were identified. Moreover, a single gene (fatty acid synthetase) that influences medium-chain fatty acids was identified.

Evaluation of a rapid protein analyzer for determination of protein in milk and cream. By Amamcharla and Metzger, page 3846. The Sprint rapid protein analyzer (CEM Corporation, Matthews, NC) and Kjeldahl method were compared for measurement of the true protein and crude protein content of milk, light cream, and heavy cream. Both methods produced similar results for true and crude protein content of milk and cream. The Sprint method was applicable for rapid and routine measurement of the protein content of milk and milk and cream. The Sprint method reduced analysis time and avoided the handling of hazardous Kjeldahl reagents.

Smallholder dairy sheep production and market channel development: An institutional perspective of rural Former Yugoslav Republic of Macedonia. By Voors and D'Haese, page 3869. Dairy sheep are important in the Former Yugoslav Republic of Macedonia. This paper analyzes the success a recently established large dairy processing company has in contracting dairy sheep farmers in 3 communities in the Former Yugoslav Republic Macedonia. Location of farms vis-à-vis collection points of the large dairy enterprise seems to be the most important choice determinant of famers to supply to this large dairy processor. Farmers preferred to sell to large dairies because of secure payments despite less transparent method of price determination. To stay in business, large processing companies could expand outreach by increasing milk production by those farmers who already supply to large dairies or by increasing collection points. 
Use of sexed semen and its effect on conception rate, calf sex, dystocia, and stillbirth of Holsteins in the United States. By Norman et al., page 3880. Sexed semen breedings of US Holstein heifers and cows were characterized by breeding year, parity, service number, region, herd size, and herd milk yield. Use of conventional and sexed semen was compared for influence on conception rate, calf sex, dystocia, and stillbirth. Conception rate decreased from 30\% with conventional semen to $25 \%$ with sexed semen for cows and from 56 to $39 \%$ for heifers, but heifer calves were produced nearly $90 \%$ of the time. Difficult births were reduced for both heifers and cows because more female calves were born, but stillbirths were reduced only for cows. 